\title{
Examining the role of individual movement in promoting coexistence in a spatially explicit prisoner's dilemma
}

Andrew E.F. Burgess ${ }^{\mathrm{a}, 1}$, Tommaso Lorenzi ${ }^{\mathrm{c}, 1}$, Pietà G. Schofield ${ }^{\mathrm{b}}$, Stephen F. Hubbard ${ }^{\mathrm{b}}$, Mark A.J. Chaplain ${ }^{\mathrm{c}, *}$

${ }^{a}$ Division of Mathematics, University of Dundee, Dundee DD1 4HN, Scotland

${ }^{b}$ College of Life Sciences, University of Dundee, Dundee DD1 4HN, Scotland

${ }^{c}$ School of Mathematics and Statistics, University of St Andrews, St Andrews KY16 9SS, United Kingdom

\begin{abstract}
The emergence of cooperation is a major conundrum of evolutionary biology. To unravel this evolutionary riddle, several models have been developed within the theoretical framework of spatial game theory, focussing on the interactions between two general classes of player, "cooperators" and "defectors". Generally, explicit movement in the spatial domain is not considered in these models, with strategies moving via imitation or through colonisation of neighbouring sites. We present here a spatially explicit stochastic individualbased model in which pure cooperators and defectors undergo random motion via diffusion and also chemotaxis guided by the gradient of a semiochemical. Individual movement rules are derived from an underlying system of reactiondiffusion-taxis partial differential equations which describes the dynamics of the local number of individuals and the concentration of the semiochemical. Local interactions are governed by the payoff matrix of the classical prisoner's dilemma, and accumulated payoffs are translated into offspring. We investigate the cases of both synchronous and non-synchronous generations. Focussing on an ecological scenario where defectors are parasitic on cooperators, we find that random motion and semiochemical sensing bring about self-generated patterns in which resident cooperators and parasitic defectors can coexist in proportions that fluctuate about non-zero values. Remark-
\end{abstract}

\footnotetext{
${ }^{*}$ Corresponding author; E-mail address: majc@st-andrews.ac.uk

${ }^{1}$ These primary authors contributed equally to this article
} 
ably, coexistence emerges as a genuine consequence of the natural tendency of cooperators to aggregate into clusters, without the need for them to find physical shelter or outrun the parasitic defectors. This provides further evidence that spatial clustering enhances the benefits of mutual cooperation and plays a crucial role in preserving cooperative behaviours.

Keywords: Spatial games, Random motion, Chemotaxis, Prisoner's dilemma, Spatial patterning

\section{Introduction}

An enduring puzzle in a wide range of biological disciplines is to identify the principles underpinning the evolution of cooperation. In this regard, much attention has been given to the prisoner's dilemma as a possible conceptual apparatus to shed some light on the way cooperative behaviours emerge and are maintained (Roca et al., 2009).

In the classical prisoner's dilemma, individuals belonging to a well-mixed population interact through a two-player game in which each individual can adopt one of two strategies: Cooperator (C) or Defector (D). The outcome of the game is determined by the following payoff matrix

$$
\begin{array}{c|cc} 
& \mathrm{C} & \mathrm{D} \\
\hline \mathrm{C} & R & S \\
\mathrm{D} & T & P
\end{array}
$$

If both players cooperate, they get the 'reward' $(R)$ payoff. If one player defects while the other cooperates, the former gets the 'temptation' $(T)$ payoff and the latter gets the 'sucker's' $(S)$ payoff. Finally, if the two players defect they both get the 'punishment' $(P)$ payoff. If $T>R>P>S$, defectors will necessarily outcompete cooperators. In fact, whether an opponent decides to cooperate or defect, the strategy D is unbeatable, by virtue of the fact that $T>R$ and $P>S$. However, if both players choose to defect they will end up with the payoff $P$, which is lower than the payoff $R$ that they would get by playing the strategy $\mathrm{C}$.

In their pioneering papers published in the nineties (Nowak et al., 1994a,b; Nowak and May, 1992, 1993), Nowak \& May developed the idea, first suggested by Axelrod (1984), of extending game theory, in general, and the standard version of the prisoner's dilemma, in particular, to include spatial interactions between the players. Using a cellular-automaton approach in 
which individual players are distributed over a two-dimensional array, they demonstrated that cooperators and defectors can coexist in the prisoner's dilemma, even if $T>R>P>S$, on condition that a spatial structure is introduced.

Several models have been considered within this theoretical framework since the original works proposed by Nowak and May [vid., for instance, Alonso-Sanz (2014); Fogarty et al. (2012); Fu et al. (2010); Gianetto and Heydari (2015); Grim (1997); Grujić et al. (2014); Kirchkamp (2000); Qin et al. (2008); Lindgren and Nordahl (1994); Nakamaru et al. (1997); Oliphant (1994); Pereira et al. (2008); Roca et al. (2009); Schweitzer et al. (2002); Szabó and Fath (2007); Vainstein and Arenzon (2001); Xia et al. (2015)]. Traditionally, explicit motion is not included in these models, with strategies moving via imitation or colonisation of neighbouring sites. More recently, increasing attention has been given to models that incorporate individual movement. For instance, Dugatkin and Wilson (1991) and Enquist and Leimar (1993) allowed individuals to migrate between patches without spatial structure. Diffusion-based dispersal of offspring was considered in Hamilton and Taborsky (2005); Koella (2000); Le Galliard et al. (2005); Van Baalen and Rand (1998). Ferriere and Michod (1995) studied an explicit diffusive process in the context of the replicator equation, and then extended their approach by including a diffusive term (Ferriere and Michod, 1996). Stochastic cellular-automaton models in which individuals can jump to a nearest empty site were developed in Jian-Yue et al. (2007); Sicardi et al. (2009); Vainstein et al. (2007). A dynamical system of reaction-diffusion type was investigated by Durrett and Levin (1994). Aktipis (2004) proposed a walk-away strategy to avoid repeated interactions with defectors. In De Andrade et al. (2009), a conditional mobility model on a lattice was presented in the context of the Chicken Game. Helbing and Yu (2008) introduced a model of success-driven migration, where individuals move to the sites with the highest estimated payoffs. Chen et al. (2011) explored the effects of mobility when individuals interact with neighbours within a prescribed view radius. The case of heterogenous view radii was analysed by Zhang et al. (2011). An aspirationinduced migration mechanism - inducing individuals to move to new sites if their payoffs are under their aspiration level - was investigated by Yang et al. (2010) and Lin et al. (2011). Meloni et al. (2009) focused on the case where individuals are situated on a two-dimensional plane, and each individual moves to a randomly chosen position with a certain speed.

To complement these earlier studies, in this paper we present a spatially 
explicit stochastic individual-based model in which pure cooperators and defectors diffuse through space and follow semiochemical cues. We believe these two generalisations to be important, because many common biological situations involve diffusion-based dispersal and/or chemotaxis guided by semiochemical gradients. In our model, individuals occupying the same position can undergo binary interactions. When interacting, they play a round of the prisoner's dilemma game, and are awarded a payoff according to their strategy. The accumulated payoff determines the reproductive fitness of individuals, and thus the number of their offspring. We investigate the case of synchronous and non-synchronous generations. Following the modelling strategy that Schofield et al. $(2002,2005)$ developed from the original approach proposed by Anderson and Chaplain (1998), we derive the individual movement rules from a system of parabolic equations describing the dynamics of the local number of individuals and the dynamics of the concentration of a semiochemical. This is a further novelty that distinguishes our work from the existing literature on spatial games.

To carry out numerical simulations, we consider a form of the prisoner's dilemma in which defectors are parasitic on cooperators - i.e., the $S$ entry of the payoff matrix (1.1) is set to zero and defectors invade a resident population of cooperators. Our main results show that allowing individuals to diffuse through space, and move up semiochemical gradients, brings about self-organised patterns in which resident cooperators and parasitic defectors can coexist in proportions that fluctuate about non-zero values. This is in stark contrast to the expected catastrophic effect that the introduction of even a small contingent of pure defectors into a population of pure cooperators would have in a well-mixed scenario. In our spatial model, coexistence has its roots firmly in spontaneous spatial organisation, without the need for individuals to remember past encounters or play elaborate strategies. This makes the results of our study applicable to a broad range of real organisms.

\section{The model}

We study the interaction dynamics between pure cooperators and defectors which move in a square domain $\Omega:=[-\ell, \ell] \times[-\ell, \ell]$. Individual movement is seen as the superposition of spatial diffusion and chemotaxis. The former is due to random motion, whilst the latter is guided by the gradient of a semiochemical emitted by individuals themselves. Individuals occupying the same position can interact with each other and the outcome 
of interactions is determined by the payoff matrix (1.1). To keep the model as simple as possible, we make the prima facie assumption that individuals cannot keep memory of past interactions. Moreover, we assume that the semiochemical is equally released from and sensed by cooperators and defectors. Despite these simplifications, the model captures a wide spectrum of biological scenarios.

\subsection{Individual movement rules}

At each time instant $t \geq 0$, the concentration of semiochemical and the number of individuals at position $(x, y) \in \Omega$ are characterised by the functions $K(t, x, y) \geq 0$ and $I(t, x, y) \geq 0$, respectively. The evolution of $K(t, x, y)$ is governed by the following reaction-diffusion equation

$$
\frac{\partial K}{\partial t}=\beta_{K} \nabla^{2} K+\nu I-\gamma K
$$

along with no-flux boundary conditions. Eq.(2.1) relies on the assumptions that the semiochemical is produced by all individuals at the same rate $\nu \geq 0$, undergoes a linear decay process at rate $\gamma>0$, and diffuses with diffusion coefficient $\beta_{K}>0$.

To describe the movement of cooperators and defectors, we make use of the following strategy:

(i) We introduce the taxis-diffusion equation below

$$
\frac{\partial I}{\partial t}=\beta_{I} \nabla^{2} I-\chi \nabla \cdot(I \nabla K)
$$

along with reflective (no-flux) boundary conditions. In Eq.(2.2), the diffusion term models the tendency of individuals to diffuse through space with motility $\beta_{I}>0$. The advection term accounts for the fact that both cooperators and defectors move up the semiochemical gradient, and the parameter $\chi>0$ is the chemotactic sensitivity coefficient.

(ii) We fix a time step $\Delta t$ and set $t_{n}=n \Delta t$, we discretise the square $\Omega$ with a uniform mesh as

$$
\begin{array}{ll}
\Delta x=\frac{\ell}{L}, & x_{i}=i \Delta x, \quad i \in[-L, L] \subset \mathbb{Z}, \\
\Delta y=\frac{\ell}{L}, & y_{j}=j \Delta y, \quad j \in[-L, L] \subset \mathbb{Z},
\end{array}
$$


and thereafter we approximate $K\left(t_{n}, x_{i}, y_{j}\right)$ and $I\left(t_{n}, x_{i}, y_{j}\right)$ by discrete values $K_{i, j}^{n}$ and $I_{i, j}^{n}$, respectively.

(iii) Following Schofield et al. (2002, 2005), we discretise Eq.(2.2) by using an explicit five-point central difference scheme to obtain the following algebraic equation for $I_{i, j}^{n+1}$, i.e., the number of individuals at grid-point $\left(x_{i}, y_{j}\right)$ at the time step $n+1$ :

$$
I_{i, j}^{n+1}=\mathcal{P}_{0} I_{i, j}^{n}+\mathcal{P}_{1} I_{i+1, j}^{n}+\mathcal{P}_{2} I_{i-1, j}^{n}+\mathcal{P}_{3} I_{i, j+1}^{n}+\mathcal{P}_{4} I_{i, j-1}^{n},
$$

where the coefficients $\mathcal{P}_{0}, \ldots, \mathcal{P}_{4}$ are given by

$$
\begin{aligned}
\mathcal{P}_{0}: \mathcal{P}_{i, j}^{n}:=1- & \frac{\Delta t}{(\Delta x)^{2}}\left[2 \beta_{I}-\chi\left(K_{i+1, j}^{n}+K_{i-1, j}^{n}-2 K_{i, j}^{n}\right)\right] \\
& \quad-\frac{\Delta t}{(\Delta y)^{2}}\left[2 \beta_{I}-\chi\left(K_{i, j+1}^{n}+K_{i, j-1}^{n}-2 K_{i, j}^{n}\right)\right], \\
\mathcal{P}_{1}: \mathcal{P}_{i-1, j}^{n}= & \frac{\Delta t}{(\Delta x)^{2}}\left[\beta_{I}-\frac{\chi}{4}\left(K_{i+1, j}^{n}-K_{i-1, j}^{n}\right)\right], \\
\mathcal{P}_{2}: \mathcal{P}_{i+1, j}^{n}= & \frac{\Delta t}{(\Delta x)^{2}}\left[\beta_{I}+\frac{\chi}{4}\left(K_{i+1, j}^{n}-K_{i-1, j}^{n}\right)\right], \\
\mathcal{P}_{3}: \mathcal{P}_{i, j-1}^{n}= & \frac{\Delta t}{(\Delta y)^{2}}\left[\beta_{I}-\frac{\chi}{4}\left(K_{i, j+1}^{n}-K_{i, j-1}^{n}\right)\right], \\
\mathcal{P}_{4}: \mathcal{P}_{i, j+1}^{n}= & \frac{\Delta t}{(\Delta y)^{2}}\left[\beta_{I}+\frac{\chi}{4}\left(K_{i, j+1}^{n}-K_{i, j-1}^{n}\right)\right] .
\end{aligned}
$$

These coefficients are proportional to the probabilities of an individual being stationary $\left(\mathcal{P}_{0}\right)$, or moving left $\left(\mathcal{P}_{1}\right)$, right $\left(\mathcal{P}_{2}\right)$, down $\left(\mathcal{P}_{3}\right)$ or up $\left(\mathcal{P}_{4}\right)$, and hence the above system may be used to generate the movement of individuals from grid-point to grid-point.

In this framework, at any step $n>1$, the algorithm for moving individuals from grid-point to grid-point is as follows:

(i) The value of $I_{i, j}^{n}$ is identified by counting the number of individuals at every grid-point $\left(x_{i}, y_{j}\right)$, and the semiochemical concentration is computed by calculating the numerical solutions of the mathematical problem defined by completing Eq.(2.1) with zero Neumann boundary conditions and suitable initial conditions. 
(ii) The probabilities $\mathcal{P}_{i, j}^{n}$ are evaluated at each grid-point by substituting the local semiochemical concentrations into Eqs.(2.6).

(iii) At each grid-point $\left(x_{i}, y_{j}\right)$, the values of the spatial-transition probabilities are used to define five intervals as

$$
R_{0}:=\left[0, \mathcal{P}_{0}\right] \text { and } R_{p}:=\left(\sum_{q=0}^{p-1} \mathcal{P}_{q}, \sum_{q=0}^{p} \mathcal{P}_{q}\right], p=1,2,3,4 .
$$

(iv) For each individual at a given grid-point, a random real number between 0 and 1 is generated, and a comparison of this number with the above ranges yields the direction of movement of the individual. Namely, the individual will not move if the random number belongs to $R_{0}$, or it will move left if the number belongs to $R_{1}$, right if the number belongs to $R_{2}$, down if the number belongs to $R_{3}$, and up if the number belongs to $R_{4}$.

\subsection{Individual interaction and reproduction rules}

We let binary interactions occur between individuals that occupy the same position. When two individuals interact, they engage in a single round of the prisoner's dilemma game and are awarded a payoff, in terms of reproductive fitness, according to their strategy.

At any step $n>1$, we allow each individual to play $M$ rounds of the game, either with the same individual or with different individuals. We assume that all individuals have the same lifetime $\tau$, and consider two possible underlying models for the reproduction rules:

(i) a synchronous model, where reproduction occurs at the end of an individual's life based on its reproductive fitness (i.e., the payoff accumulated throughout the course of previous interactions);

(ii) a non-synchronous model, where reproduction occurs at each time step with an individual producing a number of offspring equal to the integer part of its current accumulated payoff, and the reproductive fitness being then decreased by this same number.

In both cases, offspring are initially located at the same site as the parent individual, and they inherit its strategy (i.e., no mutations occur). In this setting, the constraint on the maximum number of interactions introduces an indirect limitation on the number of individuals, since it limits the potential maximum gain in reproductive fitness. 


\section{Computational simulation results}

To perform numerical simulations, we choose $\ell=100$ and use the mesh defined by (2.3)-(2.4) with $L=100$ (i.e., $\Delta x=\Delta y=1$ ) to discretise the spatial domain. Focussing on the case where a single defector invades a population of cooperators, we let the initial system to consist of 1 defector located at the centre of a randomly scattered population of $49.999 \times 10^{3}$ cooperators. Moreover, we assume that there is no semiochemical inside the system at $t=0$, i.e., we set

$$
K(t=0, \cdot, \cdot)=0
$$

The method we use to construct numerical solutions of the mathematical problem defined by endowing Eq.(2.1) with (3.1) and zero Neumann boundary conditions is based on an explicit five-point central scheme for the discretisation of the diffusion term.

We focus on a set of parameter values that is representative of an extensive range of simulation results. In particular, we choose the entries of the matrix (1.1) to have the values $R:=0.02$ and $P:=0.001$. Moreover, we set $S:=0$ to translate into mathematical terms the idea that the payoff for a cooperator against a defector is to be unproductive in terms of its contribution to future progeny. Finally, unless otherwise stated, we define $T:=0.07$. In this setting, mutual cooperators each score 0.02, and mutual defectors 0.001 . If a cooperator and a defector interact, the former scores 0 whilst the latter scores 0.07. As mentioned earlier, the setting under consideration reproduces the biological scenario in which defectors are parasitic on cooperators, since defectors invade a resident population of cooperators and $S=0$.

In analogy with the choice made by Schofield et al. (2002), we define the semiochemical diffusion coefficient $\beta_{K}:=5 \times 10^{-4}$, the semiochemical decay constant $\gamma:=1$, the chemotactic sensitivity coefficient $\chi:=2 \times 10^{-4}$, and the individual motility $\beta_{I}:=5 \times 10^{-3}$. To study the dynamics of the system with or without semiochemical secretion, we alternatively use the definition $\nu:=1$ or $\nu:=0$.

We set the number of interactions per individual per iteration $M=4$, and we define the individual lifetime $\tau:=100 \Delta t$. We select $\Delta t=0.05$ to meet the CFL condition, and thus ensure the stability of the numerical scheme. We run simulations for $5 \times 10^{5}$ time steps, which corresponds to $5 \times 10^{3}$ generations and $t \in\left[0,25 \times 10^{3}\right]$. We begin by examining the effects of semiochemical secretion and synchronous or non-synchronous generations 
on the spatio-temporal dynamics of cooperators and defectors. We will then investigate how the dynamics change in response to variations in the value of the temptation-to-reward ratio $T / R$ (i.e., the relative payoff advantage of defectors over cooperators).

\subsection{Dynamics without semiochemical secretion}

In the absence of semiochemical secretion (i.e., when $\nu:=0$ ) and with synchronous generations, the progeny of the single central defector becomes progressively organised into an almost circular expanding wavefront (vid. Fig.1). The wavefront is relatively thin, and leaves in its wake a few cooperators that coexist with a few defectors. The spatial domain remains sparsely populated. Cooperators form expanding clusters which are followed closely by flocks of parasitic defectors. The interaction between cooperators and defectors induces a severe and rapid decline in the local number of cooperators, yielding dynamic and fluctuating spatial patterns.
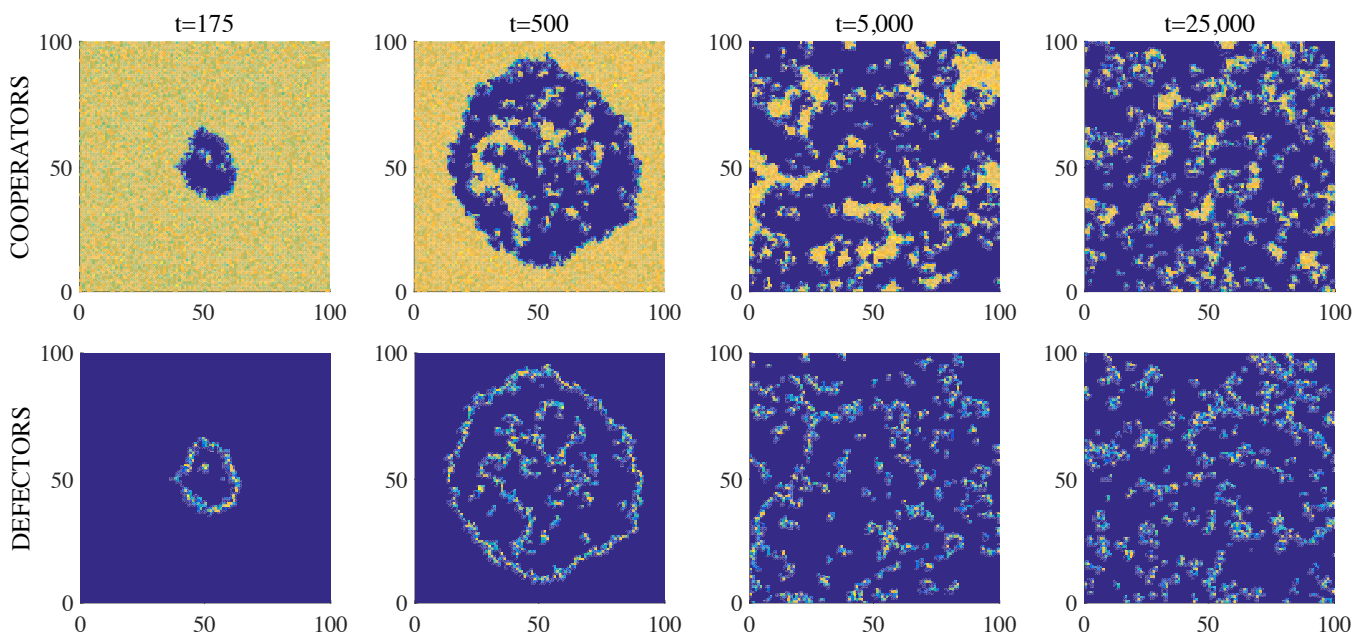

Figure 1: Snapshots summarising the time-evolution of the spatial distribution of cooperators (top panels) and defectors (bottom panels), in the absence of semiochemical secretion (i.e., when $\nu=0$ ) and with synchronous generations. The colour scale ranges from blue (low density) to yellow (high density).

As highlighted by the results presented in Fig.2, the total numbers of cooperators and defectors both fluctuate about well defined non-zero values. The time average of the total number of cooperators is $82.141 \times 10^{3}$, while that of defectors is $24.011 \times 10^{3}$. From extensive longer-term simulations 
it appears that fluctuations do not threaten the long term viability of the coexistence between cooperators and defectors. Therefore, we can conclude that the two strategies coexist in a stable way. It is worth noting that the carrying capacity of the $\mathrm{C}$ population in the absence of $\mathrm{D}$ individuals - which we estimated through additional numerical simulations carried out by letting cooperators evolve in the absence of defectors - is around a value of $336 \times 10^{3}$. The time average of the total number of cooperators reported in Fig. 2 corresponds to some $24 \%$ of this value. Hence, the proliferation of cooperators appears to be significantly limited by the presence of defectors.
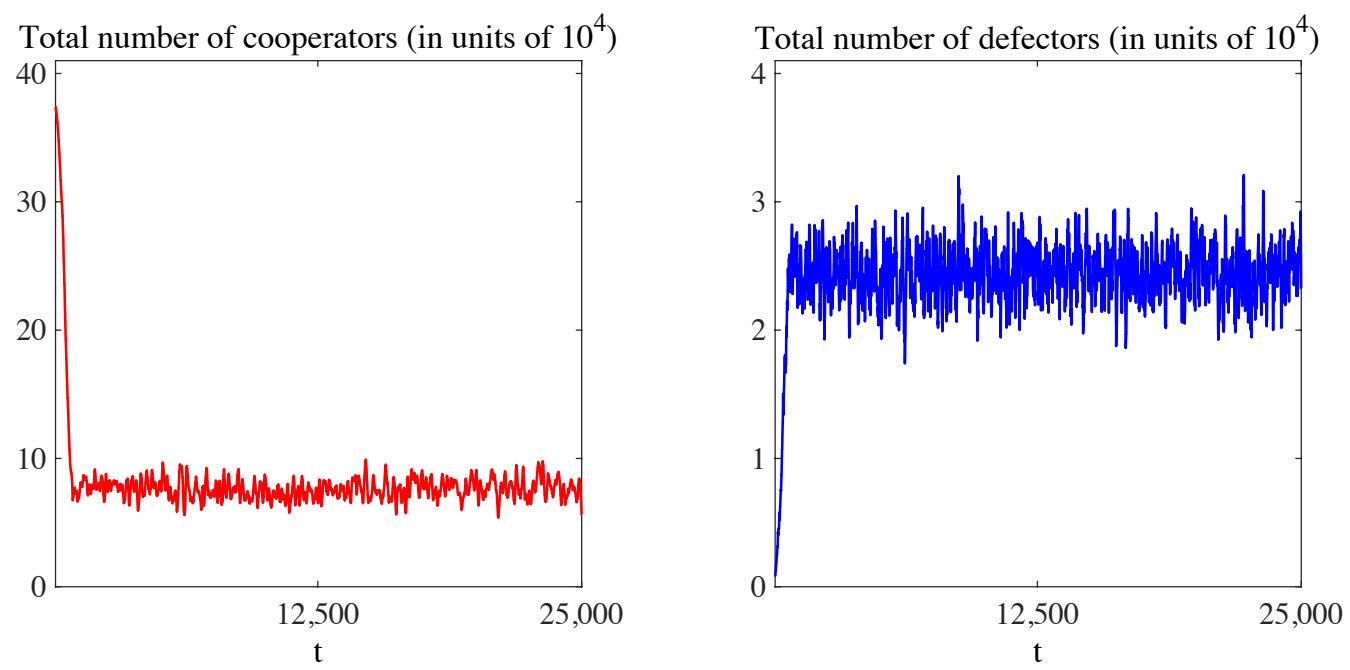

Figure 2: Plot of the total number of cooperators (left panel) and defectors (right panel) as a function of time, in the absence of semiochemical secretion (i.e., when $\nu=0)$ and with synchronous generations. The time average of the total number of cooperators is $82.141 \times 10^{3} \pm 45.46 \%$, while that of defectors is $24.011 \times 10^{3} \pm 13.91 \%$.

Analogous considerations hold when generations are non-synchronous (vid. Fig.3 and Fig.4), although the results presented in Fig.3 suggest that, compared with the synchronous case, the initial D wavefront expands faster into the $\mathrm{C}$ population. The wake of the expanding front is populated by both cooperators and defectors, which move and interact to yield a dynamic pattern studded by expanding clusters of cooperators, whose growth is curtailed by fragmented vortexes of defectors. Defectors are more sparsely distributed than in the case of synchronous generations. Furthermore, as also highlighted by Fig.10, the mean (with respect to time) of the total number of defectors is smaller compared with the case of synchronous generations, while the equi- 
valent mean becomes slightly larger for cooperators. On the other hand, the values of the standard deviations show no appreciable variations.
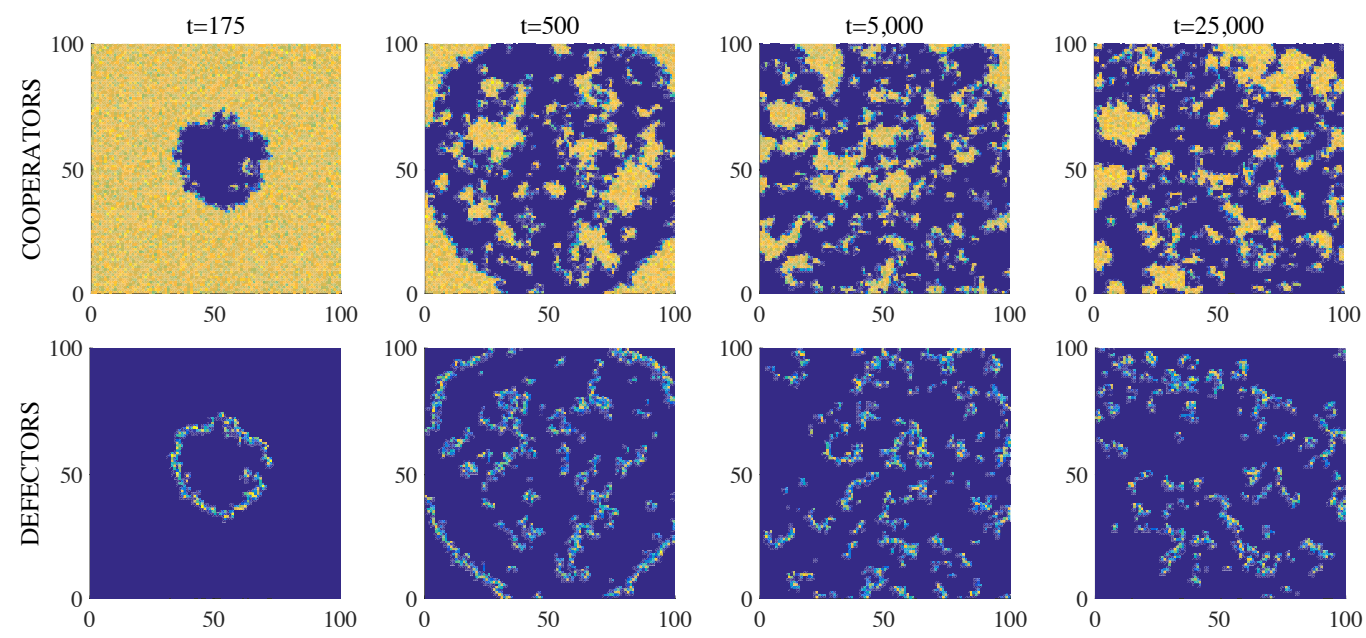

Figure 3: Snapshots summarising the time-evolution of the spatial distribution of cooperators (top panels) and defectors (bottom panels), in the absence of semiochemical secretion (i.e., when $\nu=0$ ) and with non-synchronous generations. The colour scale ranges from blue (low density) to yellow (high density).

\subsection{Dynamics with semiochemical secretion}

When semiochemical is secreted by individuals (i.e., when $\nu=1$ ) and generations are synchronous, the spatial dynamics of cooperators and defectors are similar to those observed in the case when there is no semiochemical secretion (compare the results in Fig.5 with the snapshots in Fig.1), and the two strategies coexist in a stable way (vid. the results presented in Fig.6). However, as time goes by, the clusters of $\mathrm{C}$ individuals become larger compared with the case without semiochemical secretion, while the distribution of D individuals becomes sparser and leaves a larger fraction of empty space. Although there may be algorithms and statistical methods that could allow one to quantify the clustering of the distribution of $\mathrm{C}$ individuals and the sparseness of the distribution of D individuals more formally, a precise quantification of clustering/sparseness of the spatial patterns obtained is beyond the scope of this paper.

If generations are non-synchronous, the wavefront created by the offspring of the initial defector travels outward slightly faster than in the case without 

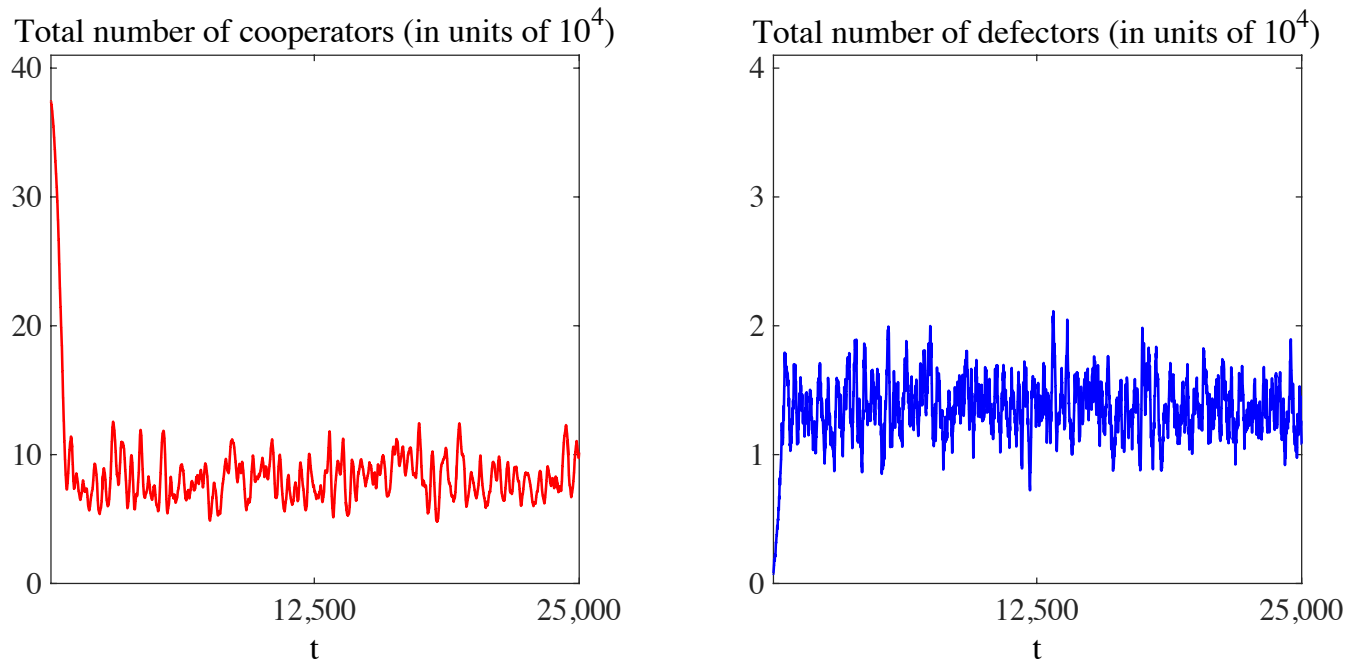

Figure 4: Plot of the total number of cooperators (left panel) and defectors (right panel) as a function of time, in the absence of semiochemical secretion (i.e., when $\nu=0$ ) and with non-synchronous generations. The time average of the total number of cooperators is $104.170 \times 10^{3} \pm 29.44 \%$, while that of defectors is $15.131 \times 10^{3} \pm 15.38 \%$.
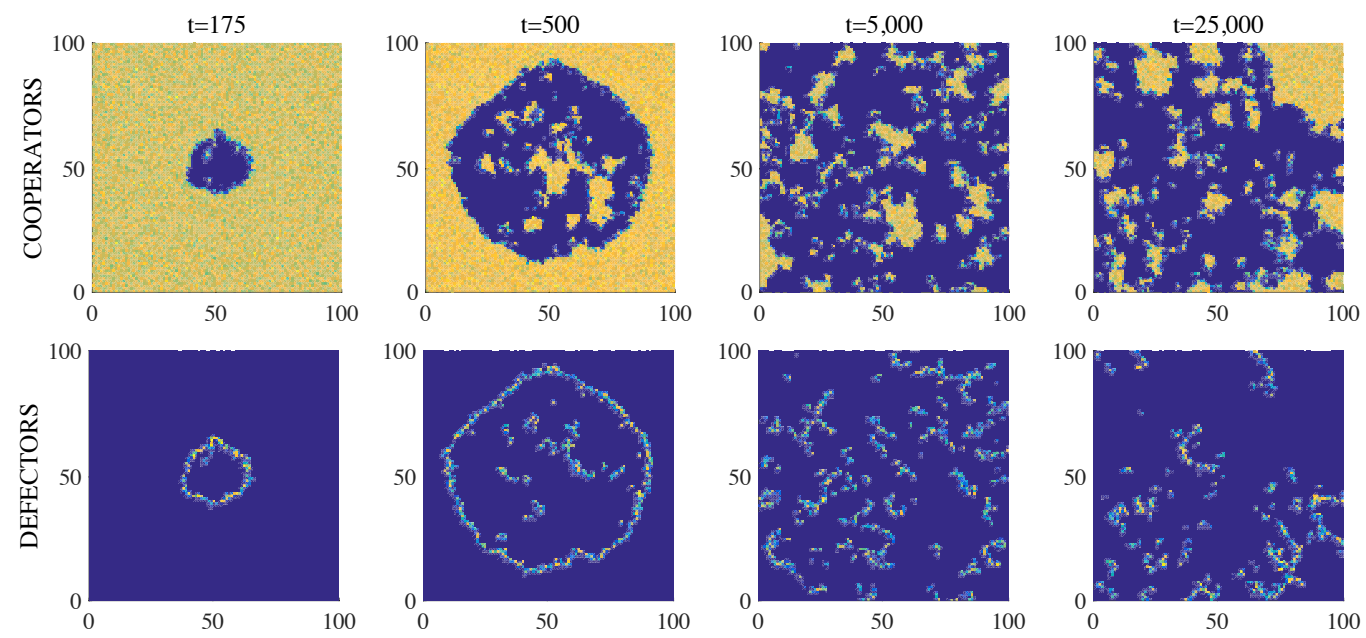

Figure 5: Snapshots summarising the time-evolution of the spatial distribution of cooperators (top panels) and defectors (bottom panels), in the presence of semiochemical secretion (i.e., when $\nu=1$ ) and with synchronous generations. The colour scale ranges from blue (low density) to yellow (high density). 

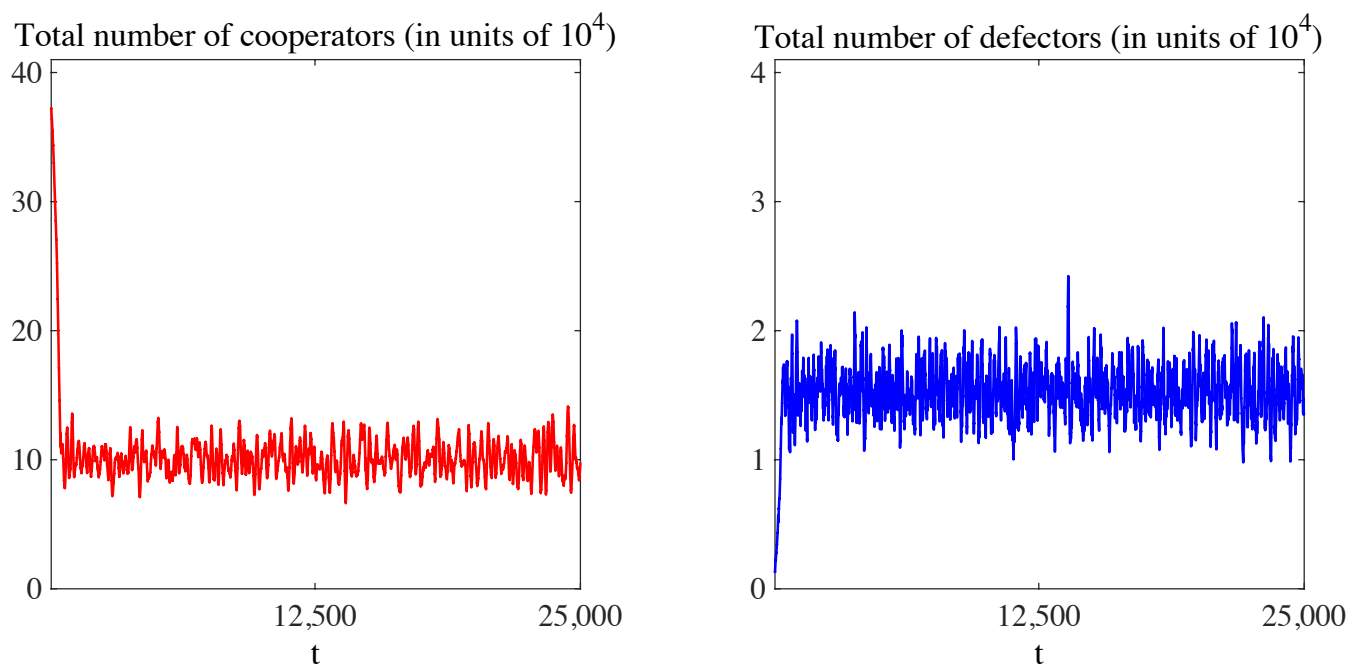

Figure 6: Plot of the total number of cooperators (left panel) and defectors (right panel) as a function of time, in the presence of semiochemical secretion (i.e., when $\nu=1$ ) and with synchronous generations. The time average of the total number of cooperators is $86.869 \times 10^{3} \pm 44.54 \%$, while that of defectors is $13.358 \times 10^{3} \pm 17.99 \%$.

semiochemical secretion. Furthermore, as depicted by the results presented in Fig.7, cooperators are distributed much more unevenly, with a smaller number of colonies of significantly larger spatial extent. The two strategies stably coexist (vid. Fig.9), although the time average of the total number of defectors is much smaller than that of cooperators and, at times, defectors are poised on the cusp of extinction. Compared with the case without semiochemical secretion, both strategies are more volatile (compare the results in Fig.9 with the curves in Fig.4). At times, when the number of defectors is particularly low, cooperators expand quickly to fill almost the entire domain before encountering small peripheral colonies of defectors, which reduce the number of cooperators back to much lower levels. Defectors form well defined filamentary wavefronts which advance in a spiral fashion altering the spatial distribution of cooperators, and annihilate each other when they meet (vid. Fig.8).

As summarised by the plot in Fig.10, compared with the case when there is no semiochemical secretion, the mean (with respect to time) of the total number of defectors is reduced - it is halved for synchronous generations and reduced by almost two-thirds when generations are non-synchronous whereas the equivalent mean becomes larger for cooperators - such an in- 

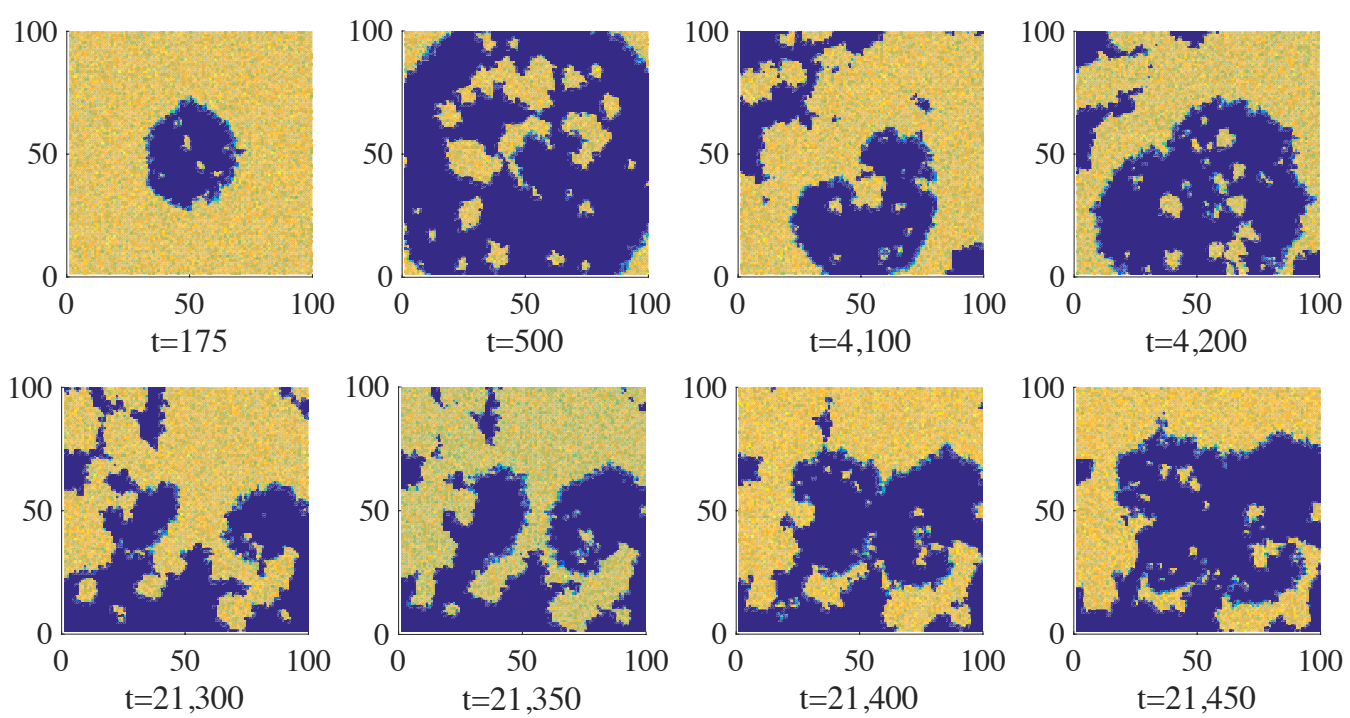

Figure 7: Snapshots summarising the time-evolution of the spatial distribution of cooperators in the presence of semiochemical secretion (i.e., when $\nu=1$ ) and with non-synchronous generations. The colour scale ranges from blue (low density) to yellow (high density).

crease is much more pronounced in the case of non-synchronous generations. On the other hand, the values of the related standard deviations remain essentially unaltered across all scenarios considered here.

\subsection{Effects of the temptation-to-reward ratio $T / R$}

To investigate how, ceteris paribus, the value of the ratio $T / R$ (i.e., the quotient between the payoff that a defector gets from the interaction with a cooperator and the payoff that a cooperator gets from conspecific interactions) impinges on the interaction dynamics of cooperators and defectors, we perform again the same simulations of the two previous subsections while holding all parameters constant except for the payoff $T$, and we record the time average of the total number of cooperators and defectors. In order to preserve the essentials of the prisoner's dilemma, we keep $T \geq R$.

The results obtained are illustrated by the plots of Fig.11, which show how the time average of the total number of cooperators and defectors varies as a function of the ratio $T / R$, in the presence or in the absence of semiochemical secretion, and with synchronous generations. Not surprisingly, for $T / R=1$ the two strategies cannot coexist, and defectors go extinct. 

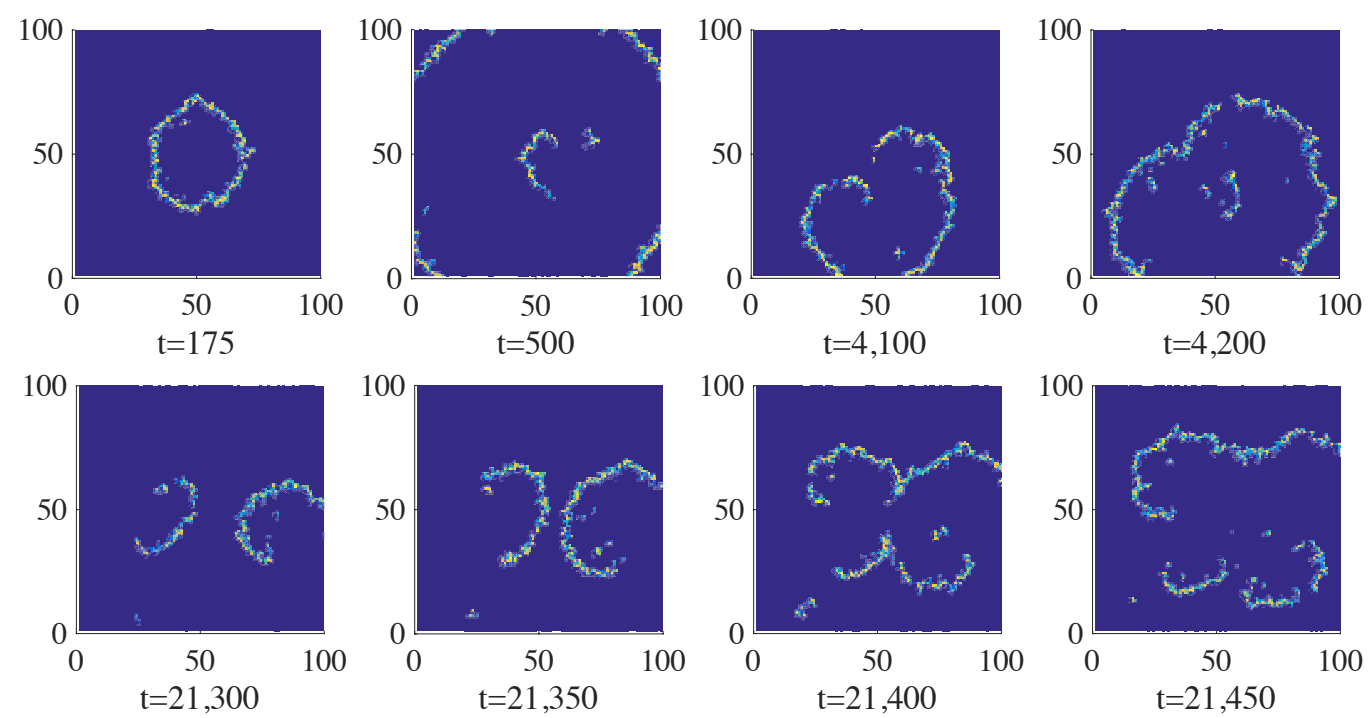

Figure 8: Snapshots summarising the time-evolution of the spatial distribution of defectors in the presence of semiochemical secretion (i.e., when $\nu=1$ ) and with non-synchronous generations. The colour scale ranges from blue (low density) to yellow (high density).

Again, for values of $T / R$ that exceed a certain threshold, all defectors die out and only cooperators survive. Between these extremes, we observe the stable invasion of a minority of defectors along with a substantial decrease in the average size of the resident population of cooperators. The limiting $T / R$ value consistent with stable polymorphism is lower in the presence of semiochemical secretion than in the case without semiochemical secretion.

The same conclusions hold for non-synchronous generations as well (vid. Fig.12). However, compared with the case when generations are synchronous, there is a narrower range of values of the ratio $T / R$ for which coexistence between cooperators and defectors occurs.

\section{Discussion and conclusions}

In the context of the prisoner's dilemma, there is now a body of evidence indicating that spatial interactions promote the coexistence of resident cooperators and parasitic defectors, in situations where the survival of one strategy would exclude the other if the interactions took place in a well-mixed scenario. Here, we have used a spatially explicit stochastic individual-based 

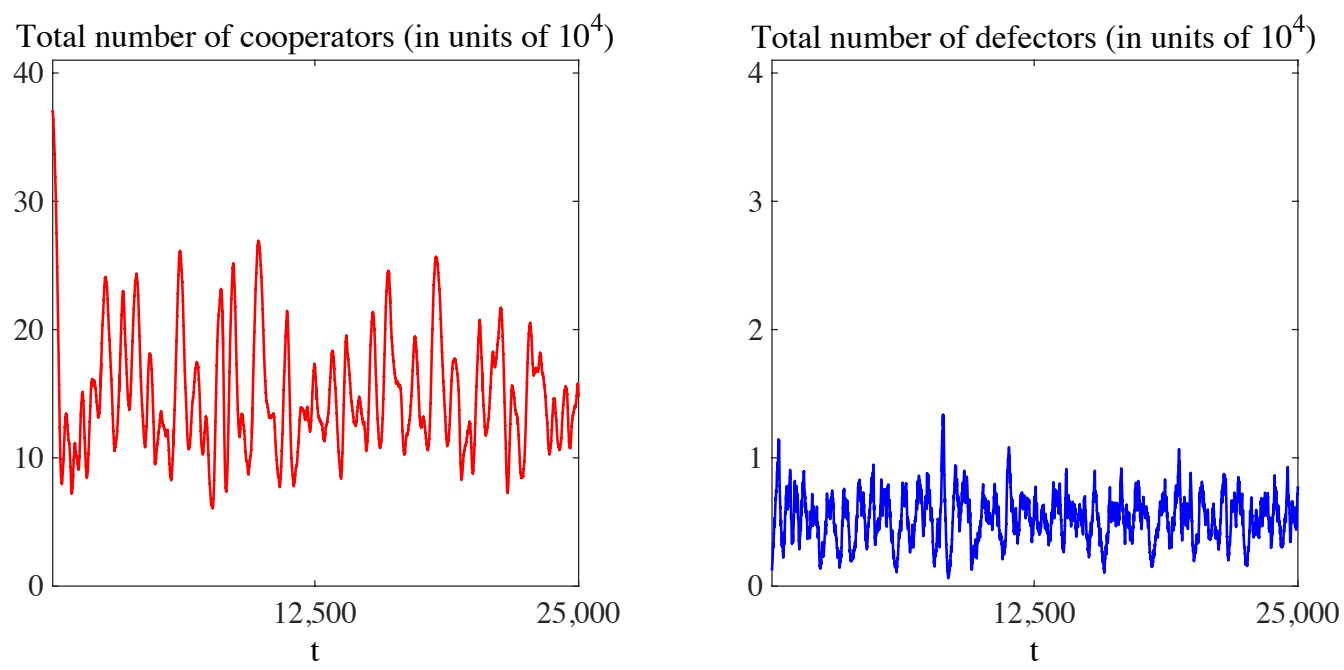

Figure 9: Plot of the total number of cooperators (left panel) and defectors (right panel) as a function of time, in the presence of semiochemical secretion (i.e., when $\nu=1$ ) and with non-synchronous generations. The time average of the total number of cooperators is $136.400 \times 10^{3} \pm 28.86 \%$, while that of defectors is $5.922 \times 10^{3} \pm 28.77 \%$.

model to investigate whether coexistence between these two strategies can occur when individuals disperse by diffusion and respond to semiochemical cues.

Our model predicts that cooperators become spontaneously organised into clusters, which minimises the potential exploitation by defectors and offers cooperators protection through spatial reciprocity. On the other hand, defectors form short filamentary structures which tend to develop into spirals while expanding. Local interactions with defectors hinder the clusters' growth and induce cooperators to migrate. In turn, the migration of cooperators forces defectors to move, as their survival depends partially on the interaction with cooperators. Iteratively, this leads to the emergence of dynamic spatial patterns in which the two strategies coexist in proportions that fluctuate about non-zero values. The essentials of these results remain intact in all the scenarios we explored (i.e., for synchronous/non-synchronous generations and in the presence/absence of semiochemical sensing).

Our in silico results agree with the in vitro results reported by Van Dyken et al. (2013) who showed that, in the context of an experimental prisoner's dilemma, spatial expansion can lead to the formation of uniform sectors of 


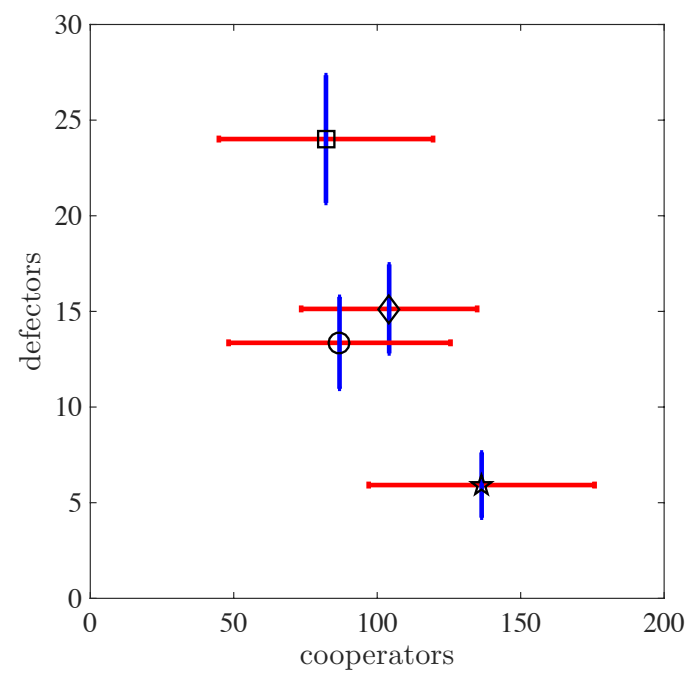

Figure 10: Plot of the average (over time) of the total number of cooperators and defectors in the four scenarios under consideration - i.e., in the absence of semiochemical secretion and with synchronous (square) or non-synchronous generations (diamond), and in the presence of semiochemical secretion and with synchronous (circle) or non-synchronous (star) generations. The blue error bars (vertical) denote the standard deviations of the defectors and the red error bars (horizontal) the standard deviations of the cooperators. All values are reported in units of $10^{4}$.

cooperators or defectors, thus reducing the direct competition between the two strategies and mitigating the selective advantage of defection.

It might be speculated that the survival of cooperators is conditioned on their capability to outrun defectors, or by the presence of physical shelters. On the contrary, in this work we have demonstrated that, even if the motility of cooperators and defectors is the same and the spatial domain is homogeneous - so that there are no physical barriers - the natural tendency of cooperators to aggregate into clusters is sufficient to preserve cooperation. These findings confirm the results presented in previous theoretical studies by Allison (2005), Durrett and Levin (1994), Fletcher and Doebeli (2009), Hauert and Doebeli (2004), Korolev and Nelson (2011), Nowak and May (1992), Ohtsuki et al. (2006), Perc and Szolnoki (2008), Santos and Pacheco (2005), Szabó and Fath (2007) and Taylor et al. (2007), and experimental works by Griffin et al. (2004), Julou et al. (2013), Kümmerli et al. (2009) and Momeni et al. (2013), and provide further evidence that spatial clustering enhances the benefits of mutual cooperation and plays a crucial role in 

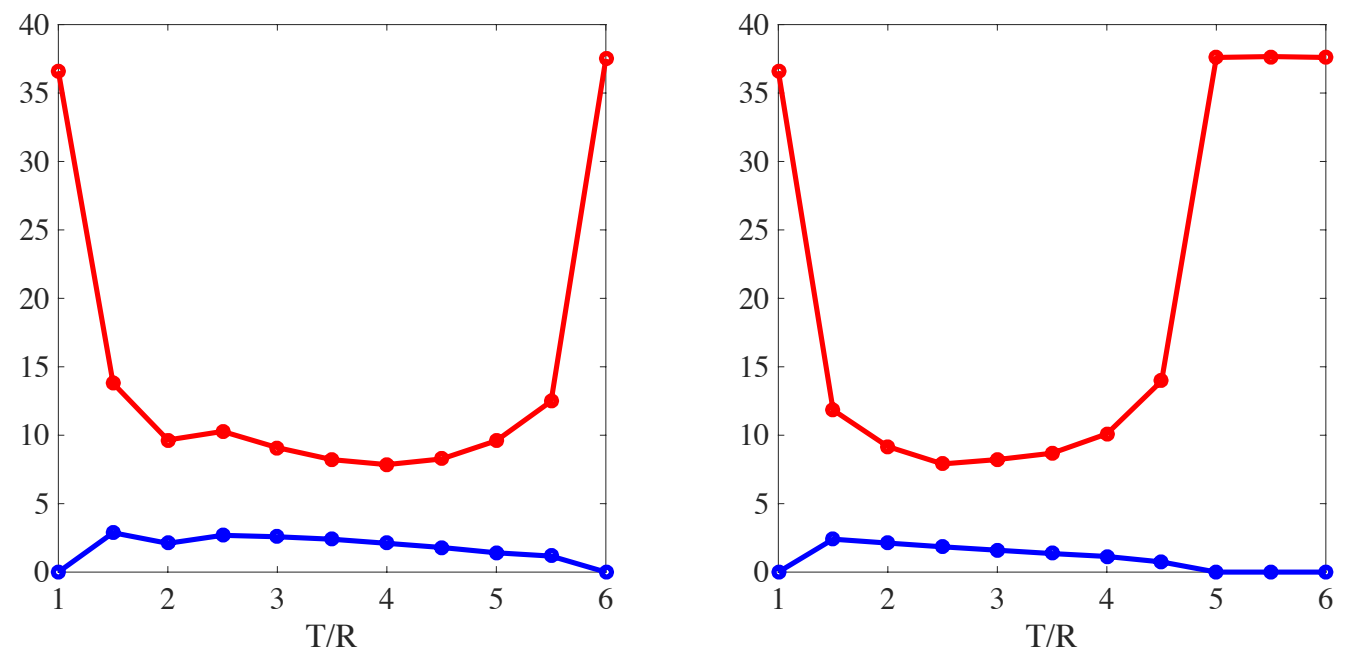

Figure 11: Plot of the time average of the total number of cooperators (red lines) and defectors (blue lines) as a function of the ratio $T / R$, in the absence (left panel) or in the presence (right panel) of semiochemical secretion, and with synchronous generations. Time averages are in units of $10^{4}$.
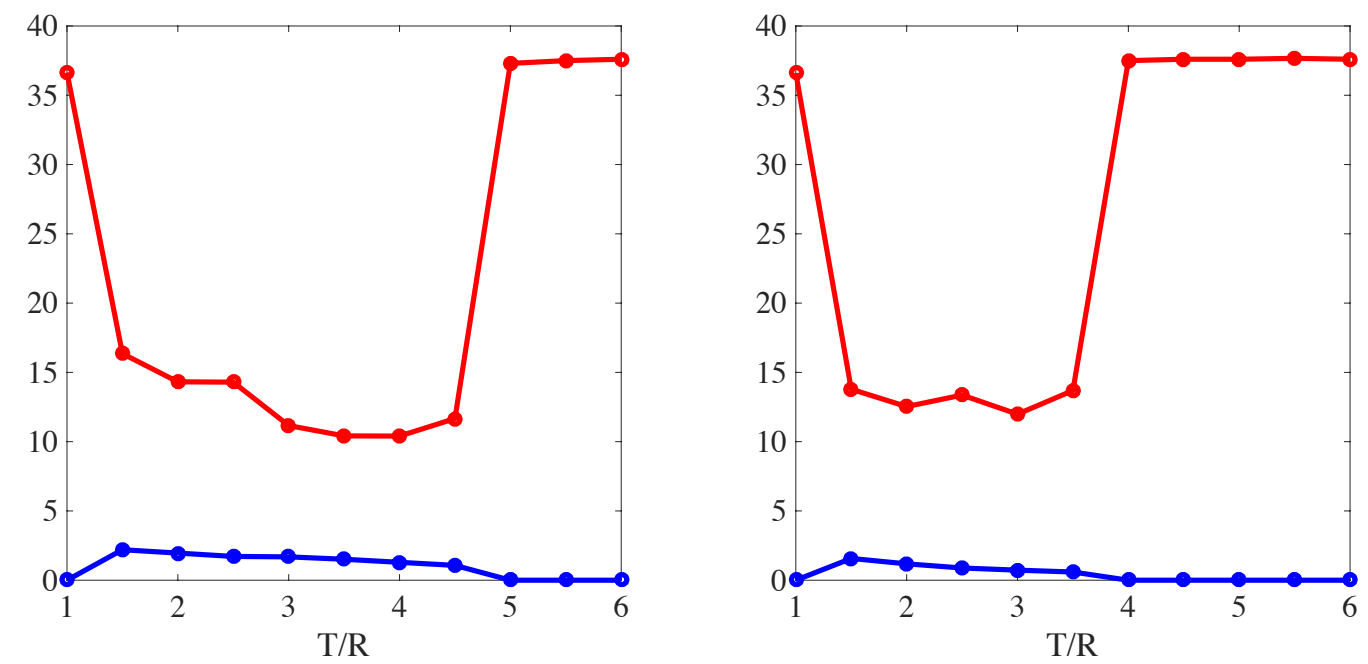

Figure 12: Plot of the time average of the total number of cooperators (red lines) and defectors (blue lines) as a function of the ratio $T / R$, in the absence (left panel) or in the presence (right panel) of semiochemical secretion, and with nonsynchronous generations. Time averages are in units of $10^{4}$. 
preserving cooperative behaviours.

The spiral structures created by defectors share some striking similarities with spiral waves arising in models of host-parasitoid dynamics, such as those reported by Comins et al. (1992), May (1995) and Solé et al. (1992). These spiral structures become more noticeable in the presence of semiochemical secretion and with non-synchronous generations, which is the case, out of those considered in our study, corresponding to the largest fluctuations in the number of cooperators and defectors. This is in line with the conclusion drawn by White et al. (1996), who suggested that the appearance of spiral waves in host-parasitoid systems correlates with more remarkable population density oscillations.

It is clear that the temptation-to-reward ratio $T / R$ is a key parameter for the coexistence between resident cooperators and parasitic defectors. As one would expect, we have found that for $T / R=1$ defectors are outcompeted by cooperators, which retain a competitive advantage by virtue of beneficial conspecific interactions. On the other hand, for intermediate values of $T / R$ defectors find a niche in which they may survive as exploiters of cooperators. More surprisingly, defectors are again outcompeted by cooperators if the value of $T / R$ overcomes a certain threshold. This is somewhat puzzling at first glance. Why do cooperators do better than defectors for high values of $T / R$ ? This can be understood by noting that, if the payoff $T$ is sufficiently higher than the payoff $R$, parasitic defectors ultimately drive the number of cooperators down to a level that cannot sustain future generations of defectors.

Compared with the case without semiochemical secretion, we have shown that the presence of a semiochemical promotes the formation of larger clusters of cooperators, and induces a reduction in the time average of the total number of defectors. This provides indirect evidence that the semiochemical may enhance the rate of conspecific interactions, and thus leads defectors to undergo a higher number of unproductive interactions. Furthermore, our simulations reveal that the semiochemical acts by shrinking the band of $T / R$ values producing polymorphisms of cooperators and defectors. This means that the chemotactic response to signalling chemicals can help resident cooperators in surviving the assault of parasitic defectors.

Taken together, these results indicate, inter alia, that the modelling framework presented here may open up new avenues of research on the role of diffusion-based dispersal and semiochemical sensing in the evolutionary dynamics of spatial games. 


\section{Acknowledgments}

AEFB gratefully acknowledges the support of an EPSRC CASE PhD studentship. The authors thank two anonymous reviewers for their helpful and insightful comments on the article.

\section{References}

Aktipis, C. A., 2004. Know when to walk away: contingent movement and the evolution of cooperation. Journal of Theoretical Biology 231 (2), 249-260.

Allison, S. D., 2005. Cheaters, diffusion and nutrients constrain decomposition by microbial enzymes in spatially structured environments. Ecology Letters 8 (6), 626-635.

Alonso-Sanz, R., 2014. A quantum prisoner's dilemma cellular automaton. In: Proc. R. Soc. A. Vol. 470. The Royal Society, p. 20130793.

Anderson, A. R., Chaplain, M., 1998. Continuous and discrete mathematical models of tumor-induced angiogenesis. Bulletin of Mathematical Biology 60 (5), 857-899.

Axelrod, R., 1984. The evolution of cooperation. Basic Books, New York.

Chen, Z., Gao, J., Cai, Y., Xu, X., 2011. Evolution of cooperation among mobile agents. Physica A: Statistical Mechanics and its Applications 390 (9), 1615-1622.

Comins, H., Hassell, M., May, R., 1992. The spatial dynamics of hostparasitoid systems. Journal of Animal Ecology, 735-748.

De Andrade, P. R., Monteiro, A. M. V., Câmara, G., Sandri, S., 2009. Games on cellular spaces: How mobility affects equilibrium. Journal of Artificial Societies and Social Simulation 12 (1), 5.

Dugatkin, L. A., Wilson, D. S., 1991. Rover: a strategy for exploiting cooperators in a patchy environment. American Naturalist, 687-701.

Durrett, R., Levin, S., 1994. The importance of being discrete (and spatial). Theoretical Population Biology 46 (3), 363-394. 
Enquist, M., Leimar, O., 1993. The evolution of cooperation in mobile organisms. Animal Behaviour 45 (4), 747-757.

Ferriere, R., Michod, R. E., 1995. Invading wave of cooperation in a spatial iterated prisoner's dilemma. Proceedings of the Royal Society of London B: Biological Sciences 259 (1354), 77-83.

Ferriere, R., Michod, R. E., 1996. The evolution of cooperation in spatially heterogeneous populations. American Naturalist, 692-717.

Fletcher, J. A., Doebeli, M., 2009. A simple and general explanation for the evolution of altruism. Proceedings of the Royal Society of London B: Biological Sciences 276 (1654), 13-19.

Fogarty, L., Rendell, L., Laland, K. N., 2012. The importance of space in models of social learning, cultural evolution and niche construction. Advances in Complex Systems 15 (01n02), 1150001.

Fu, F., Nowak, M. A., Hauert, C., 2010. Invasion and expansion of cooperators in lattice populations: Prisoner's dilemma vs. snowdrift games. Journal of Theoretical Biology 266 (3), 358-366.

Gianetto, D. A., Heydari, B., 2015. Network modularity is essential for evolution of cooperation under uncertainty. Scientific Reports 5.

Griffin, A. S., West, S. A., Buckling, A., 2004. Cooperation and competition in pathogenic bacteria. Nature 430 (7003), 1024-1027.

Grim, P., 1997. The undecidability of the spatialized prisoner's dilemma. Theory and Decision 42 (1), 53-80.

Grujić, J., Gracia-Lázaro, C., Milinski, M., Semmann, D., Traulsen, A., Cuesta, J. A., Moreno, Y., Sánchez, A., 2014. A comparative analysis of spatial prisoner's dilemma experiments: Conditional cooperation and payoff irrelevance. Scientific Reports 4, 4615.

Hamilton, I. M., Taborsky, M., 2005. Contingent movement and cooperation evolve under generalized reciprocity. Proceedings of the Royal Society of London B: Biological Sciences 272 (1578), 2259-2267.

Hauert, C., Doebeli, M., 2004. Spatial structure often inhibits the evolution of cooperation in the snowdrift game. Nature 428 (6983), 643-646. 
Helbing, D., Yu, W., 2008. Migration as a mechanism to promote cooperation. Advances in Complex Systems 11 (04), 641-652.

Jian-Yue, G., Zhi-Xi, W., Ying-Hai, W., 2007. Evolutionary snowdrift game with disordered environments in mobile societies. Chinese Physics 16 (12), 3566 .

Julou, T., Mora, T., Guillon, L., Croquette, V., Schalk, I. J., Bensimon, D., Desprat, N., 2013. Cell-cell contacts confine public goods diffusion inside pseudomonas aeruginosa clonal microcolonies. Proceedings of the National Academy of Sciences 110 (31), 12577-12582.

Kirchkamp, O., 2000. Spatial evolution of automata in the prisoners' dilemma. Journal of Economic Behavior \& Organization 43 (2), 239-262.

Koella, J. C., 2000. The spatial spread of altruism versus the evolutionary response of egoists. Proceedings of the Royal Society of London B: Biological Sciences 267 (1456), 1979-1985.

Korolev, K., Nelson, D. R., 2011. Competition and cooperation in onedimensional stepping-stone models. Physical Review Letters 107 (8), 088103.

Kümmerli, R., Griffin, A. S., West, S. A., Buckling, A., Harrison, F., 2009. Viscous medium promotes cooperation in the pathogenic bacterium pseudomonas aeruginosa. Proceedings of the Royal Society of London B: Biological Sciences 276 (1672), 3531-3538.

Le Galliard, J.-F., Ferriere, R., Dieckmann, U., 2005. Adaptive evolution of social traits: origin, trajectories, and correlations of altruism and mobility. The American Naturalist 165 (2), 206-224.

Lin, Y.-T., Yang, H.-X., Wu, Z.-X., Wang, B.-H., 2011. Promotion of cooperation by aspiration-induced migration. Physica A: Statistical Mechanics and its Applications 390 (1), 77-82.

Lindgren, K., Nordahl, M. G., 1994. Evolutionary dynamics of spatial games. Physica D: Nonlinear Phenomena 75 (1), 292-309.

May, R. M., 1995. Necessity and chance: deterministic chaos in ecology and evolution. Bulletin of the American Mathematical Society 32 (3), 291-308. 
Meloni, S., Buscarino, A., Fortuna, L., Frasca, M., Gómez-Gardeñes, J., Latora, V., Moreno, Y., 2009. Effects of mobility in a population of prisoner's dilemma players. Physical Review E 79 (6), 067101.

Momeni, B., Waite, A. J., Shou, W., 2013. Spatial self-organization favors heterotypic cooperation over cheating. Elife 2, e00960.

Nakamaru, M., Matsuda, H., Iwasa, Y., 1997. The evolution of cooperation in a lattice-structured population. Journal of Theoretical Biology 184 (1), 65-81.

Nowak, M. A., Bonhoeffer, S., May, R. M., 1994a. More spatial games. International Journal of Bifurcation and Chaos 4 (01), 33-56.

Nowak, M. A., Bonhoeffer, S., May, R. M., 1994b. Spatial games and the maintenance of cooperation. Proceedings of the National Academy of Sciences 91 (11), 4877-4881.

Nowak, M. A., May, R. M., 1992. Evolutionary games and spatial chaos. Nature 359 (6398), 826-829.

Nowak, M. A., May, R. M., 1993. The spatial dilemmas of evolution. International Journal of bifurcation and chaos 3 (01), 35-78.

Ohtsuki, H., Hauert, C., Lieberman, E., Nowak, M. A., 2006. A simple rule for the evolution of cooperation on graphs and social networks. Nature 441 (7092), 502-505.

Oliphant, M., 1994. Evolving cooperation in the non-iterated prisoner's dilemma: the importance of spatial organization. In: Artificial Life IV. MIT Press, pp. 349-352.

Perc, M., Szolnoki, A., 2008. Social diversity and promotion of cooperation in the spatial prisoner's dilemma game. Physical Review E 77 (1), 011904.

Pereira, M. A., Martinez, A. S., Espíndola, A. L., 2008. Prisoner's dilemma in one-dimensional cellular automata: visualization of evolutionary patterns. International Journal of Modern Physics C 19 (01), 187-201.

Qin, S.-M., Chen, Y., Zhao, X.-Y., Shi, J., 2008. Effect of memory on the prisoner's dilemma game in a square lattice. Physical Review E 78 (4), 041129 . 
Roca, C. P., Cuesta, J. A., Sánchez, A., 2009. Evolutionary game theory: Temporal and spatial effects beyond replicator dynamics. Physics of Life Reviews 6 (4), 208-249.

Santos, F. C., Pacheco, J. M., 2005. Scale-free networks provide a unifying framework for the emergence of cooperation. Physical Review Letters 95 (9), 098104.

Schofield, P., Chaplain, M., Hubbard, S., 2002. Mathematical modelling of host-parasitoid systems: effects of chemically mediated parasitoid foraging strategies on within-and between-generation spatio-temporal dynamics. Journal of Theoretical Biology 214 (1), 31-47.

Schofield, P. G., Chaplain, M. A., Hubbard, S. F., 2005. Dynamic heterogeneous spatio-temporal pattern formation in host-parasitoid systems with synchronised generations. Journal of Mathematical Biology 50 (5), 559583.

Schweitzer, F., Behera, L., Mühlenbein, H., 2002. Evolution of cooperation in a spatial prisoner's dilemma. Advances in Complex systems 5 (02n03), 269-299.

Sicardi, E. A., Fort, H., Vainstein, M. H., Arenzon, J. J., 2009. Random mobility and spatial structure often enhance cooperation. Journal of theoretical biology 256 (2), 240-246.

Solé, R. V., Valls, J., Bascompte, J., 1992. Spiral waves, chaos and multiple attractors in lattice models of interacting populations. Physics Letters A 166 (2), 123-128.

Szabó, G., Fath, G., 2007. Evolutionary games on graphs. Physics Reports 446 (4), 97-216.

Taylor, P. D., Day, T., Wild, G., 2007. Evolution of cooperation in a finite homogeneous graph. Nature 447 (7143), 469-472.

Vainstein, M. H., Arenzon, J. J., 2001. Disordered environments in spatial games. Physical Review E 64 (5), 051905.

Vainstein, M. H., Silva, A. T., Arenzon, J. J., 2007. Does mobility decrease cooperation? Journal of Theoretical Biology 244 (4), 722-728. 
Van Baalen, M., Rand, D. A., 1998. The unit of selection in viscous populations and the evolution of altruism. Journal of Theoretical Biology 193 (4), 631-648.

Van Dyken, J. D., Müller, M. J., Mack, K. M., Desai, M. M., 2013. Spatial population expansion promotes the evolution of cooperation in an experimental prisoner's dilemma. Current Biology 23 (10), 919-923.

White, A., Begon, M., Bowers, R. G., 1996. Host-pathogen systems in a spatially patchy environment. Proceedings of the Royal Society of London B: Biological Sciences 263 (1368), 325-332.

Xia, C.-Y., Meng, X.-K., Wang, Z., 2015. Heterogeneous coupling between interdependent lattices promotes the cooperation in the prisoner's dilemma game. PloS One 10 (6), e0129542.

Yang, H.-X., Wu, Z.-X., Wang, B.-H., 2010. Role of aspiration-induced migration in cooperation. Physical Review E 81 (6), 065101.

Zhang, J., Wang, W.-Y., Du, W.-B., Cao, X.-B., 2011. Evolution of cooperation among mobile agents with heterogenous view radii. Physica A: Statistical Mechanics and its Applications 390 (12), 2251-2257. 